\title{
A Detailed Study of EEG based Brain Computer Interface
}

\author{
Geeta Sharma, Neha Sharma, Tanya Singh, Rashmi Agrawal \\ Faculty of computer Application, Manav Rachna International \\ Institute of Research and Study, India \\ \{geetasharma.2895,nsdp8605, tani9413,drrashmiagrawal78\}@gmail.com
}

\begin{abstract}
Brain Computer Interface (BCI) generate a direct method to communicate with the outside world. Many patients are not able to communicate. for example:- the patient who are suffered with the several disease like post stroke - the process of thinking, remembering \& recognizing can be challenging. Because of spinal cord injuries or brain stem stroke the patient loss the monitoring power. EEG based brain computer interface (BCI) feature is beneficial to scale the brain movement $\&$ convert them into a instruction for monitoring. In this paper our objective is to study about various applications of EEG based signal of the different disease like spinal cord injury, post stroke and ALS (amyotrophic lateral sclerosis) etc.
\end{abstract}

Index Terms-Brain Computer Interface; Electroencephalography (EEG) signal; Amyotrophic lateral Sclerosis (ALS); Post-Stroke; Electrocorticography (ECoG).

\section{IntRODUCTION}

$\mathrm{B}$ RAIN computing interface (BCI) is a computer based technology that allows the mortal mind to communicate with electronic devices such as motorized wheelchair, robotics arm and artificial limbs without assist of any peripherals devices i.e.., nerves and muscles. The objective of $\mathrm{BCI}$ is to assess the electrical signals through the brain then amplified them and filter them to remove unwanted signals. And then convert the analogical signal to digitally signal to display on screen in the output form. Finally the digitally signal is record in a devices such as compact disk, document, floppy disk, DVD etc.

This process is occur when the different brain activities are generated through the mortal mind that will be identified by the system and translated into commands which depends on a classification algorithms.

Measuring Brain Signals:- Hans Berger who started the $\mathrm{BCI}$ invented the different electrical activity for human brain $\&$ developed. Brain waves can be evaluated with various techniques that each have advantage \& disadvantage. A commonly used techniques are:-

Electroencephalography(EEG) - EEG is used to analysis the neurochemistry in the brain through electrodes. EEG is the study of non-invasive interface that monitors the brain activities from outside the skull. It doesn't require surgery or

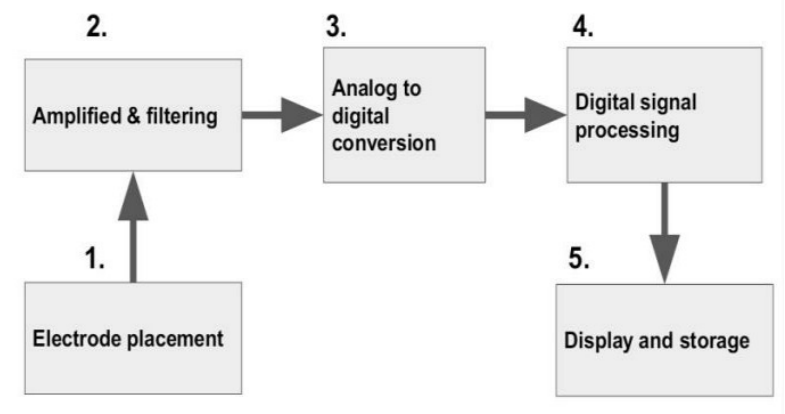

Figure 1.Brain signal recorded on the scalp through electrode, amplified and filter than it convert brain wave into machine language

permanent attachment to the device. Techniques such as computerized tomography (CT), magnetic resonance imaging (MRI), X-rays, ultrasound have all been extract the brain activity noninvasively.

EEG based BCI:- is refers to the brain activities and that considered as the Rhythmic Brain Activity. These Rhythms are pretended by distinct actions and thoughts.

EEG classified these rhythms references to their frequencies.

Delta waves:- it is our lowest level of brain wave activity. This brain signal that occur during deep dreamless sleep when the frequency up to $4 \mathrm{~Hz}$.

Theta waves:- when we are in dream, vivid imaginery \& information beyond our normal conscious awareness \& we hold our stuff fear, nightmares then theta waves are arise, in this frequency are 4 to $7 \mathrm{hz}$.

Alpha waves:- when the brain is in resting state.or in relaxation state or the frequency are 8 to $12 \mathrm{hz}$ then alpha waves signals are generated through brain.

Beta waves:-it is the best activity, present when we are alert, attention, engaged in problem solving and able to make decisions \& judgement. When frequency are 12 to 30 $\mathrm{hz}$ then beta waves signals are generated through brain. 
Table.1.brain rhythm activities with its frequencies.

\begin{tabular}{|l|l|}
\hline \multicolumn{1}{|c|}{ Rhythmic Activities } & \multicolumn{1}{|c|}{ Frequency (Hz) } \\
\hline Delta & Upto 4 \\
Theta & $4-7 \mathrm{~Hz}$ \\
Alpha & $8-12 \mathrm{~Hz}$ \\
Beta & $12-30 \mathrm{~Hz}$ \\
& \\
\hline
\end{tabular}

Electrocorticography (ECoG) - EcoG devices are embedded innermost the skull but remain outside the brain, they produce signals with better resolution as related to invasive they have reduce hazard of scar tissue. ECOG that considered cathodes located instantly on the exposed surface of the brain to store electrical activities from the cerebral. It is safer, more consistent and less technologically complicated than invasive BCI.

ECoG based BCI:- Is refers to the cursor movement that serve as the prototype for testing a new paradigms \& algorithms. While contacting the online observation of ensional cursor patients activity through one-dimcontrol which allows the participants to control either vertical or horizontal movement and through two-dimensional cursor control which enables the user to control a considerably enhanced interface. ECoG uses different activities for the cursor control testing are :-

Supplementary Motor Area (SMA) is the part of cerebral cortex especially for control of movement. SMA is the most important part of the motor imaginery based BCI application because it is based on the control of movement which is basically justified the oscillate persistent activities during the synchronized and unsynchronized movement. The activity is considered an online based measurements of brain activities.

Phase Locking Value (PLV) is an activity that can be used to analysis the performance that caused changes in long range synchronization of neural activity from EEG data. PLV measurements feature is currently established as BCI motor imaginery application.

BCI Operations:- BCI Operations are essential to record the brain signals by applying the four elements:- signals acquisition, feature extraction, feature translation \& device output through brain activities are generated and captured by the system then transmitted into commands which is considered as output.

Signal acquisition :- In signal acquisition we captured the signal from various location of the mind. it is row types of data which is meaningless.

Feature extraction :- the captured signal have noise and unwanted data. In feature extraction the noise and unwanted data are remove by using algorithms and get the desired data.

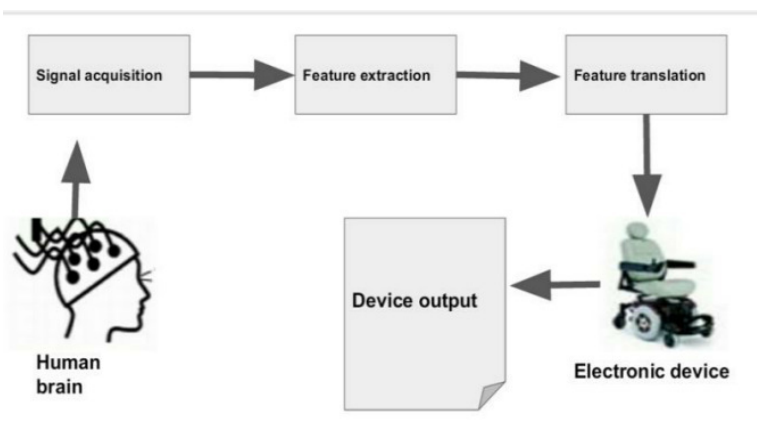

Figure 2. Mortal mind interacting with artificial device by the following different operation.

Feature translation:- The clean data converting into a command by the feature translation by the classification algorithm.

Device output:- when signals are classified the output is manipulated on the computer screen.

Problem Statement:- All of the recently BCIs subordinate under the progress of many modifications. The Problem is safety and the long-range stability of capturing cathodes utilized in invasive BCI systems remains to be determined sufficiently but not all are considered to determine fully connected with telemetry device to transfer the captured intellectual waves. Some BCI applications need an inclusive arrangements of user commands e.g., various psychological tasks, these must be detect by improving the cognitive adaptation and learning algorithms also in additional areas, such as speed, accuracy, consistency, convenience are essential for achievement of practical BCI systems.

\section{Related Study}

According to [1], The scientific intellectual that founded obvious connection \& control between the human brain and computer, known as BCI.

BCI is a computer based activity that obtain Brain signal without the help of any peripherals device such as nerves \& muscles. There are different techniques of recording signals $\&$ then apply BCI operation; signal acquisition, feature extraction, feature translation, device output by these operations electronic signals of the brain is captured over the scalp and then transmitted into commands which is considered as output that improved the task between the user and the system for that efficient BCI applications are considered that established the useful feature for the paralyzed people \& invent the electronic devices such as motorized wheelchair, robotics arms \& artificial limbs. To develop the BCI system many of the applications are working effortlessly.

According to [2], The purpose of BCI is to evaluate the electrical signals through the brain \& then amplified them and filter them to remove unwanted signals and then convert the analog signal to digital signal. This considered the real time motor imaginery process of BCI through external devices are control which is beneficial for disabled people that 
become the communication media for them. During motor imaginery the BCI is use the physiology of the nervous system which extract or classify rhythms using SVM classifier which detect the bugs and insufficient signals and converting them into desired result.

Through SVM analysis the data of motor imaginery movement of right \& left hand is classified that considered the rhythm. SVM is used to separate or identify classes, it minimize the margins to increase the generation capabilities as compared to LDA classifier which minimize distance within a class \& maximize distance between classes.

According to [3], BCI use two technique EEG and ECoG. EEG is the non invasive interface that monitors the brain activity from outside the skull. ECoG implanted inside the skull but rest outside the brain, they produce signals with better resolution as compared to invasive so, result suggested that ECoG signals has better resolution as compared to the EEG because sometimes EEG records unwanted signals that give wrong conclusion. Hence ECoG overcome the limitations of EEG. The ECoG also control the signals into cursor movement which operate tongue movement, opening $\&$ closing hand movement and the other actions, they are considered as the one dimensional or two dimensional BCI control, while experience the online observation of patients actions. The data is analyzed that helps to confirm the better adjustment of frequency bands \& anatomic location used for ECoG control of cursor movement \& final accuracy of that control.

According to [4], The different approaches and the applications are used for the functionality to generate brain signals which classified the BCI in two way.

Invasive BCI that consider as the neurosurgery because this technique consider the internal working of the brain. It is basically a kind of open surgery so it make effect on the body part.

Non-invasive BCI does not break the skin so it considered to be as compared to the invasive safest as compared to the invasive. BCI considered the real time based Applications such as prosthetic application which considered the artificial limbs. Entertainment applications that include gaming, communication through which human brain interface with the electronic devices. For the safety \& security also BCI Applications are used. BCI also faced the challenges while understanding the brain activities, capturing the signals for that BCI operations are considered through the brain signals are transmitted in the output form.

According to [5], BCI introduced the technique which are ECoG and EEG based through which many new devices are generated to control the motor disabled people activities which developed the high degree of complexity. For the evaluation of motor imaginery activity ECoG records \& analysis the observation by using BCI methodology which are Genetic Algorithm (GA), Mutual information (MI), Information Gain (IG) \& compare them with Bayesian \& KNearest Neighbor classifiers. For the high dimensionality \& outliers feature extraction Common Spatial Pattern ( CSP) used in signal processing for separating multiple observation in BCI. The comparative study of these methodology with BCI classifiers aim to develop the new communication media to the motor disabled patients.

According to [6], EEG based BCI performing the task by adopted by specific training method that based on intellectual task i.e Mutual information (MI), calculate the changes in brain rhythms. The sensorimotor rhythm (SMR), when the frequency ranges of rhythms is 13 to $15 \mathrm{~Hz}$ then it considered alpha $\&$ beta brain waves. Sensorimotor considered the stage in which the physical \& cognitive skills are developed. While calculate the observation of motor imaginery BCI different feedback moods are considered the audible \& vibractic result which produce strength or support of user task in positive way or to edify inrich effort of BCI task which consist as negative feedback. After combining the all feedback moods compare them with conventional viewable result. On the basics of compilation different session must be considered to analysis the BCI new strategies. The multiple comparison tests is considered in the sessions that improve the performance of BCI system.

According to [7], A motor imaginery BCI transmitted the control signals by applying the various algorithms which considered the rhythms according to the frequency. While measure the phase synchrony for classifying single trial EEG during motor imaginery that examine or analysis the activities of Supplementary Motor Area (SMA), is the part of cerebral cortex especially for control of movement basically justified the oscillate persistent activities during the synchronized and unsynchronized movement. The activity is considered an online based to tested the phase synchrony measurements in motor cortex for classifying EEG during motor imaginery. The phase difference between frequency $\&$ signals that generally oscillate persistent value. It is essential to test synchrony testing is known as Phase Locking Value (PLV), activity that can be used to analysis the performance that caused changes in long rang synchronized of neural activity from EEG data. The PLV measurements features is currently established as EEG motor imaginery in BCI application.

According to [8], The initial study are performing motor imagery and bCI application. In which analysis of scalp information in EEG based to left to right task in BCI. People are suffering for muscular disability who need to interact with electrical device. Most of the challenges and promises suffering people in BCI. In which used of BCI Invasive or noninvasive. Invasive BCI used open surgery in human being. It is risky for the suffering people as it is directly attach to the scalp of brain and in which used electrocortiography. Noninvasive BCI are used in attach to the outer-surface of the scalp. In noninvasive BCI used electroencepholography EEG function. BCI based on rhythms that come from motor imagination. Rhythms record the signal between $8-12 \mathrm{~Hz}$ to left to right. BCI motor imagery allow the EEG based electroencephlography used in scalp of BCI because it is not risky of the suffering people. 
According to [9], BCI is another types of field likes contribution of any medical field to suffering serious injuries. In this field such as educational system, self-configuration, marketing as different type of games and entertainment system in BCI. BCI of user and system. There are too many external-internal devices to use the muscle to complete iteration with people. In this BCI lack of challenges to restore or replace the lot of functionality. The BCI purpose involvement or analysis hand free application manage the body part and control the human behavior sending and receiving the information. In brain signal there are two types of signal are used invasive or noninvasive. Brain computer interface used noninvasive so that it is attach to the scalp. There are various challenges and application used in BCI and analysis day by day.

According to [10], BCI is a real world technique which large training or large contribution of real system. The main aims of BCI connect to the real world system increase the growth of application about helpful disable people. There are such a type of signal processing to use this technology to connect with brain signal.

Challenges of BCI to real world task:-

1. The average signal information find out the signal and capture the signal rate are increase or decrease.

2 . If the signal rate are very slow i.e. information are transfer vary slowly in the body. That's way error are generated. In the body signal are high or nor either low.

3. Autonomy:- Brain signal comes through the machine signal. In these signal are not change on the machine. He completely control and manage the body and remove the noisy data.

4. Real world system are more difficulty in imagination to task per formation, emotion, feeling, interaction with any other person. We are study on BCI to control on every days use in every stage and situation

According to [11], Brain computer interface connection of brain and machine device to communication with externally and internally control by the signal. It is fluctuate the voltage between neurons and signal applier. Invasive BCI are direct connected to the scalp that's way invasive signal are higher quality of signal but it is risky for human. Noninvasive used EEG or MEG magnetic resonance imaging. EEG used in scalp of outside the brain. It is attach to the wire in in the device to controlling the robots, games movement of cursor etc. The channel are select EEG signal. This means BCI are signal equitation of EEG or MEG then select the useful signal and transfer the noise signal then feature extraction the classification of the signal. Now a days we are study of the many technology remove unwanted and noisy signals.

According to [12], Machine learning is a priority of preprocessing technology and EEG base of BCI. This BCI takes minimum time and gives the communication speed to 6-8 per minute. In the EEG analysis online separation of electrical signals. The current argumentation between two function. Now we are study of EEG based signal. Brain computer interface communication with brain signal, from muscles and peripheral device. Signals are fluctuate the according voltage to the user of user device. Machine learning is also analysis of emotion, sleep stage, feeling, through etc.

According to [13], BCI task with the paralyzed people. It use the noninvasive of EEG based to the signal.it provide multidimensional movement to manage the body part through which the brain. Noninvasive BCI used in scalp activity to provide the signal point to point the control injuries. The research is the main motive of the provide the communication with the suffering people. It is provide the communication in two types of invasive or noninvasive. Noninvasive used in scalp of EEG based and invasive is open surgery. It has provide high speed of the communication. It is a clinical risk or infected of body.

According to [14], EEG and motor imagery in real world to communicate with other. In which the classification between EEG and BCI. This paper describe by various algorithms in real time testing. Brain computer interface work with left and right hand movement and these frequency are 70 to $95 \%$. It store the information to provide faster feedback. Rapid prototyping analysis of update very fast technique application to design the signal. These are flexible to store the information.

According to [15], Here we learn about EEG based BCI Application for stroke rehabilition by robotics arms. Stroke is a big injury by which many other problems face by the patients like voice, focusing and sensibility. Rehabilation help for monitoring \& communicating with daily life for patients. Rehabilation is better then manual therapy due to its instants improvements in patients movements activities. BCI helps for monitoring the external devices \& systems like computer, switches and robotic wheelchair. Functional Electric Simulation(FES) of BCI, is utilized for hand activities. In FES we study on two exercise first quadriplegia patients that want to grip a glass of water by using BCI FES device and other one is a stroke patients. The youth mind is very strong \& its memorize power is high so after the stroke problem it can take action by the action of damaged, injured area. The memorizing power is called as neuro-plasticity which focus on every location where command is given from one part to another. We find out the chances of using non-invasive BCI \& robotic rehabilation for post-stroke rehabilition. This technique is applied for converting the fantasy into the instruction by acquiring the signal from scalp that recorded by eeg. it gives better performance and accurate result. We are also study about translate synchronization to asynchronous role of motor vision where object are controlled by artificial limbs.

According to [16], While analyzed the classification algorithms for generate BCI system of EEG algorithms that recognize the brain activity and amplified them after that break down them into different ranges according to signal are generated. BCI have five types of classification algorithm that are linear classifiers, neural networks, non-linear, Bayesian classifiers, nearest neighbor classifiers \& combina- 
tion of classifiers. The SVM are usable for synchronous BCI. The $\mathrm{k}$ nearest neighbor(KNN) algorithm \& mahalonobis distance algorithm are used for nearest neighbor classifiers. Their are many other classification techniques that not use today's day for objective of BCI. If BCI explore more in clinical activities, new technologies will be applied into the uses. these techniques are perform on the large amount data sets of EEG signal.

According to [17], The normal users can simply monitoring the robots by using the keyboard, mouse, joystick but its complex to abnormal or paralysis patient to control. these some interfaces sip \& puff system, single switches and eye -tracking systems are help for those people. But these technique do not applied on other injured patient such as amyotrophic lateral sclerosis (ALS), multiple sclerosis (MS) and stroke. Still autonomous robots were not useful for transfering the particular location for the muscular disable people that's why these type of interfaces or medium are generated for those patients who can not control the computer or system.

EEG is the part of non-invasive interface of brain computer. There are various application of eeg signals like-controlling a moving cursor on the screen, selection of letters from a virtual keyboard, playing games, pattern recognizing etc.

The EEG based robots are control by the mind signal. Brain controlled manipulators \& mobile robots these are the two types of mind controlled robots. A FRIEND system generate by the Graser et al. Which is the manipulator. Noninvasive BCI access the many types of mind signal likeelectroencephalograms(EEG), magnetoencepalogram (MEG), blood-oxygen-level dependent(bold) signals, but eeg is more demanding because of low cost.

According to [18], As we can communicate with the outside world but many patient not able to communicate.

For example:- the patient who cause the several disease like post-stroke, the process of thinking, remembering \& recognizing can be challenging because of spinal cord disease or brain stem stroke, the patient loss the monitoring power.

The clinical application of neurorehabilitation based on brain computer interface on EEG for monitoring motor refit. It helpsfor monitoring the patient which have amyotrophic lateral sclerosis \& spinal cord disease \& stroke.many features like sensorimotor rhythms (SMR), slow cortical potentials(SCP), SMR'r SCP's based system use the factor like frequency or time domain. The motive of clinical application pof bci is useful for patient have disease like amyotrophic lateral sclerosis(ALS), several CNS damage such as spinal cord injuries \& stroke and epilepsy and attention deficit \& hyperactive disorder (ADHD). Result is high shortage in controlling \& motor function.

BCI gives choice \& complementary monitoring control \& motor control refit \& down-regulation of cortical activity. it provides neural network communication with the device to motor control \& full movement of damage area. the cortical physiology that confirm the way in which human brain or mind understand.

According to [19], Electroencephalography (EEG) based on BCI can read the people consideration, thought \& thinking for motor vision. The device applicable for disable people by reading the brain of people which exist left $\&$ right imaginary movement \& judge the actual movements. Kalman filter of BCI's is applied for signal manipulation to selection of the efficient signal. the common spatial pattern(SCP) and radial Basis function(RBF) are the classification method that used for feature extraction. The common spatial pattern(CSP) is applied in channel selection. The best channel picking on the basis of maximum vector derived from CSP. The complex Morlet wavelet tranform technique used in alpha \& beta rhythms. It discover \& sorted the left \& right movement imagination. The feature subset selection algorithm for feature extraction \& multilayer backpropogation neural network for the classification Increase the efficiency of the classification of motor imagery left and right hand movement by using the kalman filter.

According to [20], BCI is a mediator between mortal mind \& system. Electroenceplograpgy (EEG) is applied for better performance \& effective or accurate classification. Sparse representation is applies for mental task classification.this algorithm is divided into two classes according to the BCI data. Common spatial pattern(CSP) was a big tool for using the dictionary. Differential dictionary that introduce by Zhou et al. Which is basis on the minimization objective function renovate \& differential technique occur in objective function. Mostly sample of sparse representation are zero by this it provide the accurate result. It provide separability \& adaptivity. sparse representation is better to other classifiers support vector machine(SVM) and $\mathrm{K}$ nearest neighbor(KNN) Because kernel in SVM and neighbor in KNN are difficult.

BCI Based Algorithms :- During motor imaginary, the BCI uses the physiology of the nervous system which are classified and extract the rhythms based on their frequencies. The Classification algorithms used to design BCI system by considering the BCI Operations. For the BCI classification algorithms different classifiers is used to extract the signals in the commands.

Linear Classifier :- the linear classifier are based on the different classifiers algorithms which are

Linear Discriminant Analysis (LDA) :-

is a method used in pattern recognition \& machine learning to find a linear combination of features that separates two or more classes of objects or events. It minimize distance within a class \& maximize distance between classes. The procedure commonly used for multiple classes is the 'One Versus The Rest'(OVR) which exist in separating each class from all the others. This procedure has a very low proportional obligation which makes it convenient for online $\mathrm{BCI}$ be system. This classifier is simply to use \& commonly produce good results. LDA classifier be goes with asynchronous BCI \& has been used with prosperity in a excellent 
number of BCI system such as motor imaginery based BCI. The disadvantage of LDA is it's straightness that produce inferior results as compared to nonlinear EEG data.

Support Vector Machine (SVM):-

This method is also used to separates or identify classes. By minimize the distance from the nearest point \& maximize the margins to increase the inference techniques. It's capacity to manage the high Spatial obstacles allaying the effect of ' the curse of dimensionality'. It can be used for both linear \& nonlinear problems by using the 'kernel trick' which escape the explicit mapping that is needed to get linear learning algorithm with radial basis function. Kernel is generally used in SVM to identify the distance between two Vectors.

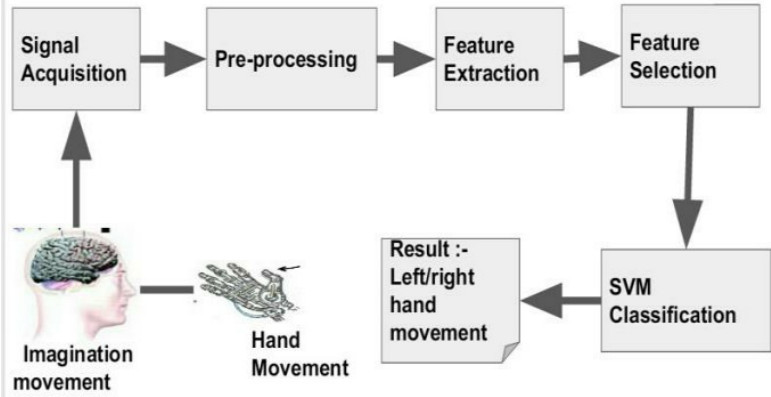

Figure 3. BCI monitoring the hand movements by applied the SVM classifier.

Nonlinear classifier:- It initiate Bayesian classifiers used for Bayes quadratic has been applied for BCI, it is not represented here as it is not conventional, presently, persistent sufficient for real-time BCI. This classifiers generate nonlinear decision boundaries. It is originated which permits them to operate further effectual alienation of unreliable patterns than discriminative classifiers. However, these classifiers are not as popular as linear classifiers or Neural Networks in BCI applications.

Bayes quadratic:- Bayesian classification objective is allotting a element vector to the class it exists the highest opportunity. The Bayes rule is applied to evaluate the subsequent opportunities that a element vector has belonging to a given class using the MAP (Maximum A Posteriori) rule, the class of this element vector can be considered Bayes quadratic include a different ordinary grouping of data that control quadratic decision boundaries. This classifier is not popularly used for BCI, but include in success to motor imagery and mental task classification.

$\mathrm{K}$ Nearest Neighbor(KNN):- $\mathrm{K}$ nearest neighbor(KNN) is the non-parametric method which is applied for the classification.it is used for statistic calculation and pattern recognition. The input have the adjacent example of the $\mathrm{k}$ and the output is the class membership like any other classifier or decision tree. Object is classified by the majority of vote of its class neighbor an $\mathrm{k}$ refer to the number of neighbor which we consider and it is a positive number typically small.
If the $\mathrm{k}=1$ then variable is allocate to the class of the closest element it has.

Neural network:- neural network are work with linear classify. In which several artificial activities works to recall the brain signal. These algorithm are work on EEG (electroencephalogaphy), to take short time classifier given to the brain. Neural network are applied in standard multilayer perceptron (MLPs) methods classified to the brain activities. These network are applied to compare FIR (finite impulse response) to clear purifying the processing of signals.

\section{Conclusion \& Future Study}

BCI is majorly used in science history which provide functionality between mind and gadget which consider the many application for further use transmission tool for severe disability and artificial limbs to monitoring disabilities such as quadriplegic, cerebromedullospinal. Neurorehabilition after neurological injuries and entertainment \& gaming. Polygraph test, brain fingerprinting, trust assessment. BCI is a field of practical study so it always in it's infancy. The comparative research on various components of $\mathrm{BCI}$ is ongoing that investigate useful instinctual waves; signal capturing, feature extraction and translation methods for combining short- and long-range adaptations between human and device to improve the performance that are suitable for BCI applications such as clinical applications; communication, movement control, Neurorehablitation.

\section{ACKNOWLEDGMENT}

We, student of FCA completed this paper under the guidance \& supervision of Dr. Rashmi Agrawal and Dr. Prasanjit Banerjee Accendere KMS pvt. Ltd.. Their contribution are sincerely appreciated and gratefully acknowledged.

\section{REFERENCES}

[1] Joseph N. Mak [Member, IEEE], Jonathan R.Wolpaw,Albany, Clinical Applications of Brain-Computer Interfaces: Current State and Future Prospects

[2] Roxana Toderean 1, Iuliana Chichisan.Application of Support Vector Machine for the Classification of Sensorimotor Rhythms in Brain Computer Interface

[3] Eric C. Leuthardt, Kai J. Miller, Gerwin Schalk, Rajesh P. N. Rao, and Jeffrey G. Ojemann.Electrocorticography-Based Brain Computer Interface-The Seattle Experience

[4] Aroosa Umair, Ureeba Ashfaq, and Muhammad Gufran Khan.Recent Trends, Applications, and Challenges of BCI

[5] Aswinseshadri. K Dr.V. Thulasi Bai. feature selection in brain computer interface using genetics method

[6] I. N. Angulo-Sherman and D. Guti'errez,. Effect of different feedback modalities in the performance of brain-computer interfaces

[7] Yijun Wang, Bo Hong*, Xiaorong Gao, and Shangkai Gao.Phase Synchrony Measurement in Motor Cortex for Classifying Single-trial EEG during Motor Imagery

[8] Lei Qin, Lei Ding And Bin He. Motor imagery classification by means of source analysis for brain computer interface applications.

[9] Sarah N. Abdulader*, Ayman Atia, Mostafa_sami M. Mostafa. Brain computer interface: Applications and challenges

[10] Melody M. Moore. Real word application for brain computer Interface technology.

[11] School of computer engineering, kiit university, bhubaneswar India. Brain computer interface issues on hand movement 
[12] Klaus Robert Miiller Michael Tangermann.Machine learning for real time signal trial EEG analsis from brain computer interfacing to mental state monitoring.

[13] Jonatha R-wolpaw*and dennis j.mcfaxland. Control of a twodimensional movement signal by a noninvasive brain computer interface in human

[14] Christoph guger, alo is schlogl, chritra newper. Rapid prototyping of an EEG based brain computer interface BCI

[15] Chuanchu Wang,Kok Soon Phua, Kai Keng Ang, Cuntai Guan, Haihong Zhang, Rongeseng Lin.A feasibility study of non-Invasive Motor imaginery. BCI based robotic rehabilitation for stroke patients
[16] Fabien lotte, Marco Congedo, Anatole Lecuyer, Fabrice Lamarche, Bruno Arnaldi. A review of classification algorithm for eeg based brain computer interface.

[17] Luzheng Bi, Member, IEEE, Xin-An Fan, and Yili Liu,Member, IEEE.EEG-Based brain -controlled mobile robots: A survey.

[18] Sergio Machado, Leonardo ferreira Almada and Ramesh Naidu Annavarapu. Progress \& prospective in EEG-based brain computer interface: clinical application in neurorehablitation.

[19] Nik Khadijah Nik Aznan,Yeon-Mo Yang. Applying kalman filter in eeg based brain computer interface for motor imagery classification.

[20] Rasool Ameri, Aliakbar Pouyan,Vahid Abolghasemi. EEG signal based on sparse representation in brain computer interface application. 